\title{
Real-time Urban Population Monitoring Using Pervasive Sensor Network
}

\author{
Gautam S. Thakur ${ }^{1}$, Teja Kuruganti ${ }^{1}$, Miljko Bobrek ${ }^{1}$, Stephen Killough ${ }^{1}$, \\ James Nutaro ${ }^{1}$, Cheng Liu ${ }^{1}$, Wei $\mathrm{Lu}^{2}{ }^{{ }_{\phi \phi}}$ \\ ${ }^{1}$ Oak Ridge National Laboratory, 1 Bethel Valley Road, Oak Ridge, TN 37831 USA, \\ ${ }^{2}$ Uber Advanced Technologies Center, Pittsburgh, PA \\ ${ }^{1}\left\{\right.$ thakurg, kurugantipv, bobrekm, killoughsm, nutarojj, liuc\}@ornl.gov, ${ }^{2}\{$ luweift\}@gmail.com
}

\footnotetext{
${ }^{\phi \phi}$ A section of this work has been done while Dr. Wei Lu worked at the Oak Ridge National Laboratory, USA. No additional work was conducted by Dr. Lu after he joined Uber Advanced Technologies Center.
}

\begin{abstract}
It is estimated that $50 \%$ of the global population lives in urban areas occupying just $0.4 \%$ of the Earth's surface. Understanding urban activity constitutes monitoring population density and its changes over time, in urban environments. Currently, there are limited mechanisms to non-intrusively monitor population density in real-time. The pervasive use of cellular phones in urban areas is one such mechanism that provides a unique opportunity to study population density by monitoring the mobility patterns in near real-time. Cellular carriers such as AT\&T harvest such data through their cell towers; however, this data is proprietary and the carriers restrict access, due to privacy concerns. In this work, we propose a system that passively senses the population density and infers mobility patterns in an urban area by monitoring power spectral density in cellular frequency bands using periodic beacons from each cellphone without knowing who and where they are located. A wireless sensor network platform is being developed to perform spectral monitoring along with environmental measurements. Algorithms are developed to generate real-time fine-resolution population estimates.
\end{abstract}

\section{CCS Concepts}

- Information systems $\rightarrow$ Geographic information systems $\quad$ Hardware $\rightarrow$ Wireless integrated network sensors - Computer systems organization $\rightarrow$ Real-time system architecture

\section{Keywords}

Population dynamics; transportation; spectral monitoring; sensors.

\section{INTRODUCTION}

The central observation that must be made to characterize urban activity is the presence and movement of large numbers of people $[1,2]$. Urban activity monitoring in real-time requires understanding population density and its changes over time. This information is required for modeling mobility, which is an important measure to evaluate efficiency, accessibility, and

Permission to make digital or hard copies of all or part of this work for personal or classroom use is granted without fee provided that copies are not made or distributed for profit or commercial advantage and that copies bear this notice and the full citation on the first page. To copy otherwise, or republish, to post on servers or to redistribute to lists, requires prior specific permission and/or a fee.

SIGSPATIAL'16, October 31-November 03, 2016, Burlingame, CA, USA ACM 978-1-4503-4589-7/16/10

DOI: http://dx.doi.org/10.1145/2996913.2996937 livability in urban areas. Present approaches based on static data (e.g., census and surveys) to monitor population density and its variations are typically crude or have sharp limits to practical application. By definition, static data does not provide information about dynamics, except in the most limited sense of portraying trends over years or decades. While it is possible to interpolate between infrequent data points, these guesses are necessarily inaccurate and, it is impossible to determine the range of uncertainty in any useful way. Other approaches to monitoring population density involve collecting data directly from the phones of cell phone subscribers using call detail records (CDR). This is the primary, existing technology for continuous monitoring of population density in urban areas. However, tracking location (or any other data) obtained from cell phones is highly intrusive. To acquire information about population density in this way requires either unannounced mining of data obtained from cellular service providers, which poses obvious privacy concerns and consequent unacceptable risks to the business of the service provider. Additionally, "opt-in" tracking programs, which have relatively low rates of participation and are likely to be biased towards particular types of users. There are also passive cell phone tracking approaches based on receiver power measurements, but these typically have short ranges, limited to a few hundreds of feet indoors. There are more advanced detectors that, within a given area (e.g., shopping mall), detect the cell phone activities to understand shopping behaviors but these detectors primarily use $\mathrm{Wi}-\mathrm{Fi}$ signals and require user's mobile phone to be connected to the public Wi-Fi. Newer approaches to estimating population density and mobility have been developed using social media data analysis [3]. These techniques are promising for determining urban activity, but are mostly applicable to large event monitoring where many social media users are sending messages specific to event activities and timelines. This approach is less useful for normal day-to-day urban activity monitoring because of the relatively low volume of geotagged data compared to the number of cell phone users at large. Since use of social media data also raises privacy concerns, access to individual messages is often limited by social media services. Other population density and mobility approaches are based on usage of public transportation [5], but they lack the granularity of individual population and suffer from the limitation that normal public transportation patterns may be disrupted during special events and/or emergency response scenarios. As a result, lack of a non-intrusive real-time population density monitoring, impacts important applications such as ambient population modeling (LandScan[1]) and transportation mobility modeling[2]. 


\begin{tabular}{|c|c|c|c|c|c|}
\hline \multirow{8}{*}{ 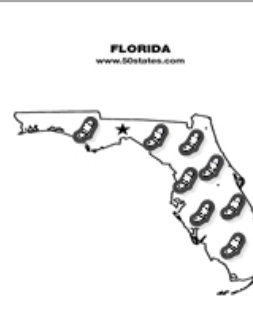 } & Region & Population & Time & \multirow{9}{*}{ 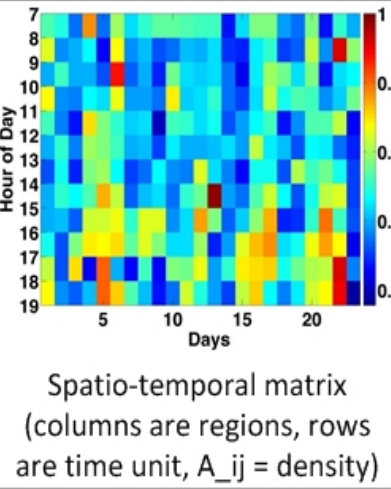 } & \multirow{9}{*}{$\begin{array}{c}\text { High-resolution spatio- } \\
\text { temporal population } \\
\text { mapping }\end{array}$} \\
\hline & Geo-1 & 100 & 8:00-9:00 AM & & \\
\hline & Geo-2 & 150 & 1:00-2:00 PM & & \\
\hline & Geo-3 & 200 & 3:00-4:00 PM & & \\
\hline & Geo-4 & 500 & 7:00-8:00 PM & & \\
\hline & ......... & $\ldots$. & $\ldots$ & & \\
\hline & $\ldots \ldots \ldots$ & $\ldots$. & $\ldots$ & & \\
\hline & Geo-n & 90 & 4:00-5:00 PM & & \\
\hline $\begin{array}{c}\text { Cellphone sensing } \\
\text { through human } \\
\text { mobility }\end{array}$ & \multicolumn{3}{|c|}{$\begin{array}{c}\text { Estimating } \\
\text { Population Density } \\
\text { Numbers (PDN) }\end{array}$} & & \\
\hline
\end{tabular}

Figure 1: Passive sensing workflow

These applications currently use static population density data obtained from various sources like Census, satellite imagery, etc., which do not represent the real-time aspect of the population density changes, allowing for significant uncertainty in long-term observations. Availability of such real-time data will create new opportunities in urban activity modeling.

The proposed research creates a system for continuous, nonintrusive monitoring of population density in urban environments and use this data to generate realistic models of ambient population and mobility. The proposed approach avoids problems of privacy associated with intrusive tracking technologies, used presently to estimate population density, while still enabling the accurate, high frequency estimates that have been demonstrated with the intrusive approaches. The system observes spectral and environmental activity in urban areas by passively capturing LTE signals. This is achieved through a radio frequency antenna tuned to cell phone frequency bands that is used for monitoring location beacons sent by cellular handsets to towers. A developed software for analysis of cell tower downlink data is used to extract the number of users in the cellular area. These numbers are down sampled to generate fine-resolution population estimates.

One important application of our approach is harnessing the capability of sensing human activity to drive ORNL's LandScan efforts, in near real-time modeling of ambient population and discovering land use information. Currently, LandScan uses static spatial data and imagery analysis technologies and a multivariable dasymetric modeling approach to disaggregate census counts within an administrative boundary. The proposed approach will provide a unique capability to capture near real-time human mobility patterns at a much finer granularity than aggregate census data and density distribution to monitor population dynamics. In addition, the number of sensors deployed in a geographical region can be tuned as required, thereby enhancing the spectral monitoring and sensing to capture spatial data from social economic boundaries rather than administrative boundaries.

Studies have shown that spatio-temporal longitudinal analysis of human mobility demonstrates predictable and stable behaviors in visiting locations at a particular time. For example, people go to their jobs during the daytime and stay at home in the evenings. Our data collection method will spur research in spatio-temporal modeling and categorization of human mobility patterns to enable the discovery of land use information (such as residential, commercial, public and government locations). LandScan and other population or modeling applications will benefit from this data by automating the categorization of land use into commercial, residential, and mix land, which in turn will be used for used for urban planning, energy infrastructure planning, and first response activities.

Urban transportation systems support human activities and population mobility and are major factors in energy consumption and environmental emission problems. Maintaining the transportation system's efficiency during both normal activity and special events is an important issue that betters understanding of populations in motion will help to resolve. Data from this system will also serve to improve accuracy of population mobility modeling in transportation systems.

This proposal seeks to develop a technology capability that is accurate, practical, and expandable for providing population density data (along with environmental data) that will be foundational to the field of urban activity monitoring. The proposed technology will allow researchers to make precisely quantifiable statements about the motion and distribution of the population. By providing a solution to this fundamental problem, the proposed research is an indispensable key to understanding cities and the populations that define them [8,9]. The proposed approach will immensely benefit, in addition to the two applications discussed above, a host of other applications like local response, safety personnel, public safety, and disaster response based on the information received in real-time. In particular, real-time data collection and monitoring will help assess critical situations and coordinate responses for evacuation management. In addition, our approach will help enable efficient deployment of necessary resources and enable rescue team to prepare and quickly react to unforeseen events.

\section{APPROACH}

Real-time urban population monitoring using pervasive sensor network approach is shown in Figure 1. It consists of four main steps. In first step, hardware platform is developed and deployed that enables passive sensing of cellphone in the region of interest. In this work, we have utilized LTE system to sense cellphone users. In second step, the resulting signal is translated into Population Density Numbers using an open source library that output approximate number of cellphones detected in the range of interest. In third step, gridded spatio-temporal clustering (approximately in $153 \mathrm{~m} \times 153 \mathrm{~m}$ block) is performed to measure the distribution of cellphone users during a typical day. Finally, in fourth step, fine-resolution population data is produced using dasymetric modeling.

Traditional passive cell phone tracking uses power level monitoring in bands of interest and limited in accuracy of estimating counts and range. We propose to exploit LTE system 


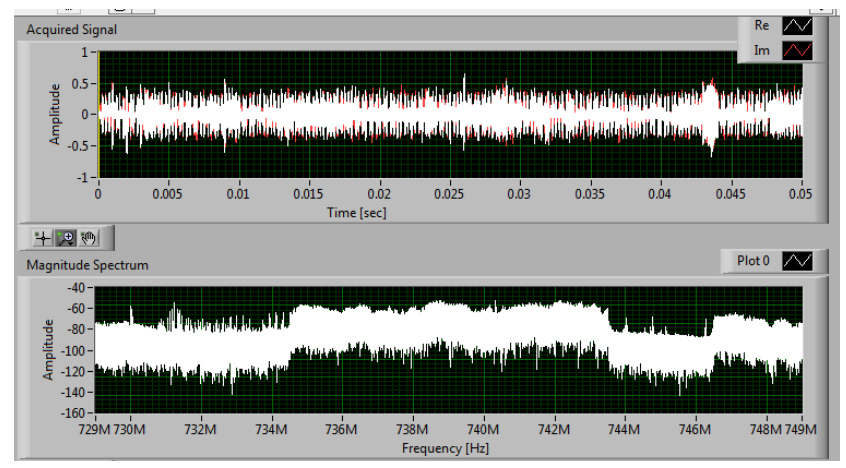

Figure 2: 5-frame long recording of the signal

synchronization which occurs in different phases of establishing and maintaining the link between the Base Station (BS) and the Subscriber Units (SUs). LTE downlink from BS to SU includes resource allocation to users within BS range. Each SU entering into a cell will be assigned a Cell Radio Network Temporary Identifier (C- RNTI), which reveals no private user information but is used to identify the user for time- frequency slot scheduling. Data from the downlink channel is analyzed using software based on open source libraries, LTEye[3], to generate the number of unique users over time. This software utilizes LTE control channel data, which contains meta-data for uplink and downlink transmissions, without accessing any private user data or encrypted LTE channels. Our prototype hardware/software platform performs the following:

- Acquire the RF signal from the cell towers in the area of interest, and down-convert it to the quadrature $\mathrm{I}$ and $\mathrm{Q}$ baseband samples using USRP N210 radios[4].

- Correlate the I and Q samples with three Primary Synchronization Signals (PSS) and 168 Secondary Synchronization Signals (SSS) to recover the LTE PHY parameters for the particular cell tower.

- Use as input PHY parameters and I/Q samples to the LTEye software to decode Physical Control Format Indicator Channel (PCFICH) and detect how many symbols (CFI) are allocated for Physical Downlink Control Channel (PDCCH) in every frame. Subsequently decode the PDCCH channel and extract the Cell Radio Network Temporary Identifiers (C-RNTI) in every frame.

The temporal C-RNTI data is analyzed to obtain temporal analytics. Example in Figure 2 shows $50 \mathrm{~ms}$ of data to identify number of unique C-RNTIs (indicating number of users). The 5frame long recording identified 387 unique users operating simultaneously. The software demonstrates the feasibility of generating real-time data that can be used towards understanding the dynamics of urban activity. The hardware device output approximate number of cellphones detected, which will be fed into the population database that is part of the GIS system software as described in the next section.

\section{CONTROLLED EXPERIMENT}

A controlled experiment was carried out in ORNL premises to validate the proposed method. The analysis output of 51 seconds of data at 1-second intervals to identify number of unique CRNTIs (indicating number of users). During the 51 seconds there were 300 unique users identified in the cell area, while their location in the area is unknown. The developed method demonstrates the feasibility of generating real-time data that can be used towards understanding population distribution. Next steps include, optimization of software and hardware to reduce computational complexity using custom-built RF hardware for bands of interest and FPGA-based preprocessing of the signal.

\section{ESTIMATE POPULATION}

We apply simple moving average technique to smooth out the variation between sensed data and calculating observed population counts per unit time. If $\lfloor\kappa\rfloor$ is data rate at which asynchronous queue is filled-up by sensor data, then the moving average rate calculation $v$, such that $v \geq\lfloor\kappa\rfloor$ for non-null average calculation. The moving average use micro-batch size $m$ for next aggregate raw cellphone population number such that $m$ is filled by $\lfloor\kappa\rfloor$ in the next moving average calculation iteration. Also, our experience has shown that population spikes rarely occurs and the ebb and growth usually happens over a longer period of time (linear increase and decrease). A non-weighted moving average per unit time for a $k$-size micro-batch for a window of size $n$, the population calculation is defined by $p_{L}, p_{L-1}, p_{L-2}, \ldots$, $p_{L-(n-1)}$. The overall average calculation for the window size $n$ will then be

$$
U S P=\frac{p_{L}, p_{L-1}, p_{L-2}, \ldots, p_{L-(n-1)}}{n}=\frac{1}{n} \sum_{i=0}^{n-1} \mathrm{p}_{\mathrm{L}-1}
$$

The future values will be calculated using the micro-batch size and old batch will get replaced by the new values as

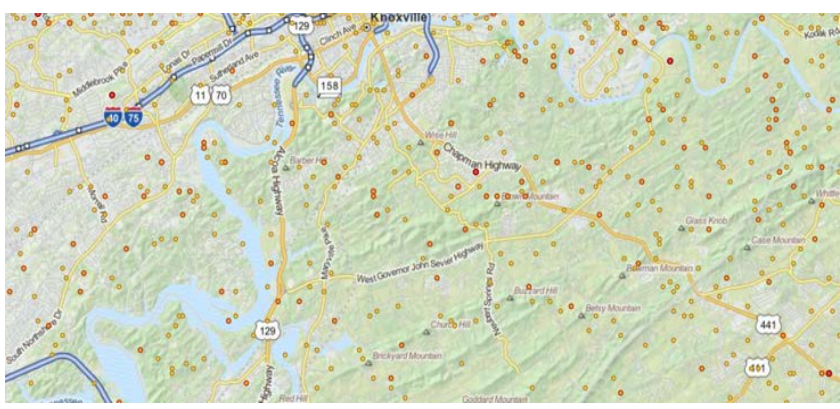

Figure 3 Fine-resolution population distribution

$$
U S P_{\text {now }}=U S P_{(\text {now }-1)}+\frac{p_{l+w}}{n+w}-\frac{p_{l-w-n}}{n+w}
$$

The period to generate the moving average is tunable, however it should be greater than $\mathrm{N} 210$ sensing time unit. Our experiments have shown that $\mathrm{N} 210$ can be set for split seconds sensing, thus allowing moving average window to be of arbitrarily large size (few seconds to a minute). For the purpose of this study, our sensor was able to monitor cellphones every few seconds and therefore, we kept our moving average calculation for one-minute time window.

\section{POPUlATION DISTRIBUTION MODEL}

We want to show a high-resolution down-sampled distribution of population in space and time. There are two prominent methods used - Dasymetric modeling, which produces a high-resolution population estimates by utilizing information from source population distribution and underlying geographic layers. This approach identifies boundaries and as well as captures non-linear distribution of population densities for the given region. In contrast, Ariel interpolation downscales coarse-scale geographical data from origin to a high-resolution destination scale. However, Ariel interpolation assumes random boundaries and dasymetric interpolation assumes linear or smooth distribution. In this work, we use Dasymetric approach for its ability to include ancillary information, however the choice of methods for generating fine- 
resolution population distribution can vary depending upon the need and accuracy. We have used Intelligent Dasymetric mapping method technique for generating fine-resolution population distribution[6]. The technique use source population $P o p_{s}$ and downscales to target population $\widehat{P O P}_{t}$ as

$$
\widehat{P o p}_{t}=\operatorname{Pop}_{s} \frac{w_{t}}{\sum_{t \in s} w_{t}}
$$

where $w_{t}$ is the observed population count in target region $t$. This population count is generated through regression analysis of the population and ancillary layers. A sample snapshot is shown in Figure 3 for the city of Knoxville, USA.

\section{CONCLUSION}

This work has demonstrated a new capability for continuous, nonintrusive monitoring of population density in urban areas. This capability was achieved through a system that discovers cellular phones in an urban area by monitoring power spectral density in cellular frequency bands using periodic beacons from each cell phone without knowing who and where they are located.

The sensing is carried out using a radio frequency antenna tuned to cell phone frequency bands that monitors location beacons sent by cellular handsets to towers. We have exploited LTE system synchronization, which occurs in different phases of establishing and maintaining the link between the Mobile Base Station (BS) and the Subscriber Units (SUs). The synchronization is based on Zadoff-Chu sequences to generate synchronization signals and preambles such as Primary Synchronization Signals (PSS), Sounding Reference Signals (SRS), random access preamble (PRACH), uplink control channel (PUCCH), and uplink traffic channel (PUSCH). This method allows to identify the unique signatures of each cell phone thereby accurately estimating number of cell phones in a given region.

Population density numbers are generated after translating sensed signals into aggregate population counts. Using Dasymetric projections, we down-sample these numbers to generate a fineresolution population distribution.

Experimental testing has shown that the proposed approach is accurate, practical, and expandable for generating population density data that will be foundational to the field of urban activity monitoring. In addition, this approach provides a real-time assessment of population and their mobility patterns.

In the future, we want to develop fine-resolution population models using ancillary information from buildings, settlements, and facility land use. We would like to includes these population projections into main stream population models such as LandScan to better understand the mobility patterns. We hope the proposed method will assess critical situations and coordinate responses during emergency and evacuation management as well as enable efficient deployment of necessary resources.

\section{ACKNOWLEDGMENTS}

This manuscript has been authored by UT-Battelle, LLC under Contract No. DE-AC05-00OR22725 with the U.S. Department of Energy. The United States Government retains and the publisher, by accepting the article for publication, acknowledges that the United States Government retains a non-exclusive, paid-up, irrevocable, world-wide license to publish or reproduce the published form of this manuscript, or allow others to do so, for United States Government purposes. We are thankful to Budhendra Bhaduri for his support during research work.

\section{REFERENCES}

[1] B. Bhaduri, E. Bright, P. Coleman, and M. L. Urban, "LandScan USA: A high-resolution geospatial and temporal modeling approach for population distribution and dynamics," in GeoJournal, 2007, vol. 69, no. 1-2, pp. 103-117.

[2] H. L. Lu Wei, Liu Cheng, Bhaduri Budhendra, Thomas Neil, "A Global System for Transportation Simulation and Visualization in Emergency Evacuation Scenarios," 2014.

[3] S. Kumar, E. Hamed, D. Katabi, L. Erran Li, S. Kumar, E. Hamed, D. Katabi, and L. Erran Li, "LTE radio analytics made easy and accessible," in Proceedings of the 2014 ACM conference on SIGCOMM - SIGCOMM '14, 2014, vol. 44, no. 4, pp. 211-222.

[4] ETTUS, "USRP N210," USRP N210 Kit (USPR N200, 2 SMA-Bulkhead RF Cables, Ethernet Cable, Power), 2016. [Online]. Available: https://www.ettus.com/product/details/UN210-KIT.

[5] Wikipedia, “ $\{$ Geohash $\}--\{$ W $\}$ ikipedia $\{$,$\} The Free$ Encyclopedia." 2016.

[6] N. N. Nagle, B. P. Buttenfield, S. Leyk, and S. Speilman, "Dasymetric Modeling and Uncertainty.," Ann. Assoc. Am. Geogr., vol. 104, no. 1, pp. 80-95, Jan. 2014. 